\title{
Accelerated Machine Learning as a Service for Particle Physics Computing
}

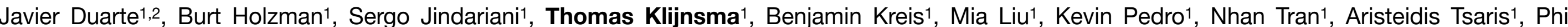

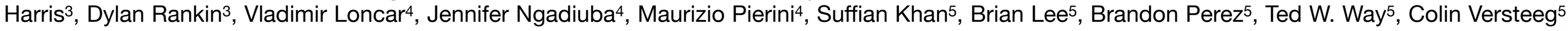
Scott Hauck ${ }^{6}$, Shih-Chieh Hsu6, Matthew Trahms ${ }^{6}$, Dustin Werran ${ }^{6}$, Zhenbin Wu ${ }^{7}$

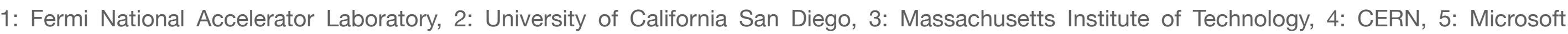
6: University of Washington, 7: University of Illinois Chicago

FERMILAB-POSTER-19-143-E

complexity of data

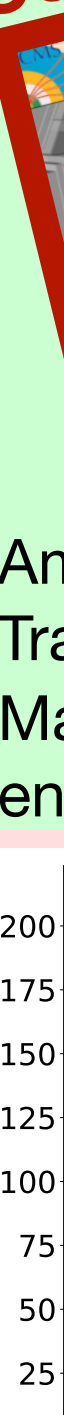

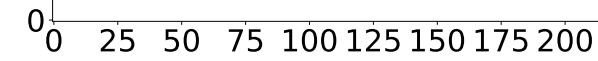

QCD enough

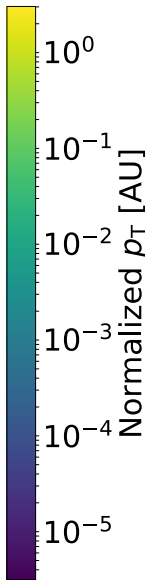

(averages of 5000 images)

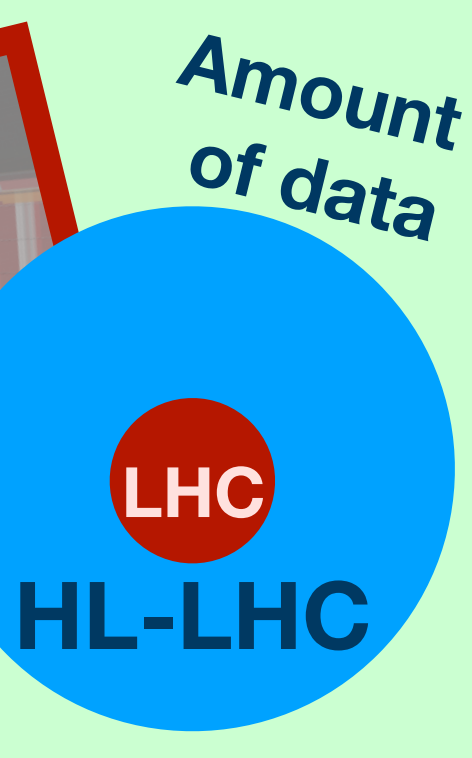

Inference-as-a-service

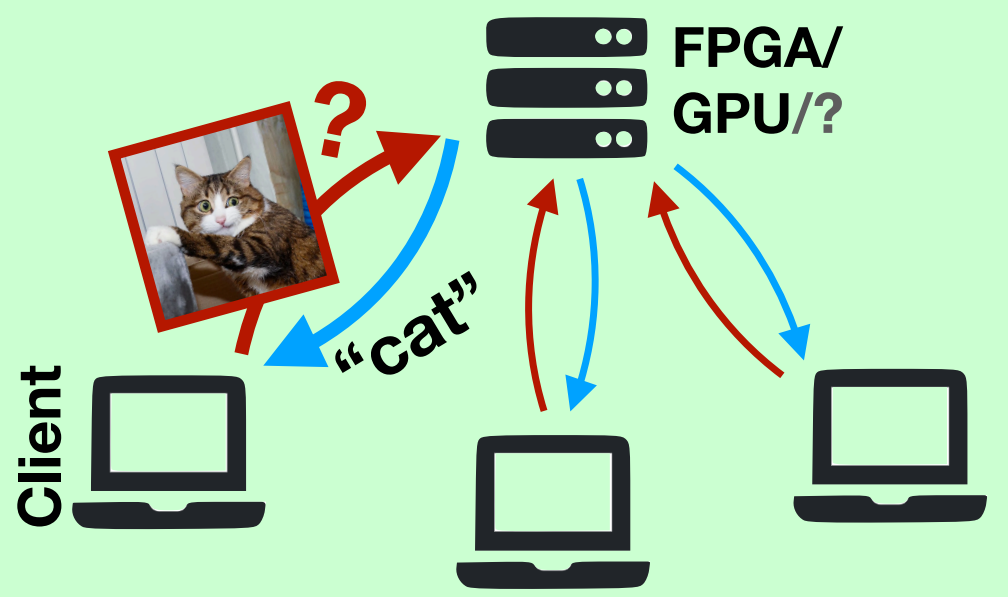

- Amount and complexity of high-energy physics data increases dramatically from 2025 onward

- Traditional algorithms will require too much CPU time

- Machine learning can solve combinatorially-scaling problems in constant time, but must be fast

\section{Inference-as-a-service}

$\mathrm{VM}$ in Microsoft Azure central US Cloud Service
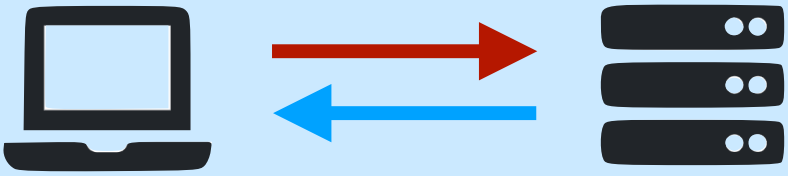

60-80 ms Optimal case: $10 \mathrm{~ms}$

CPU @ Azure Data Box FNAL
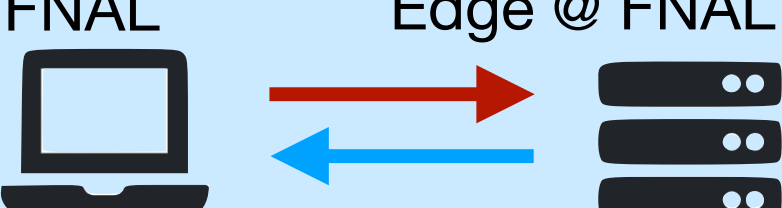

$20 \mathrm{~ms}$

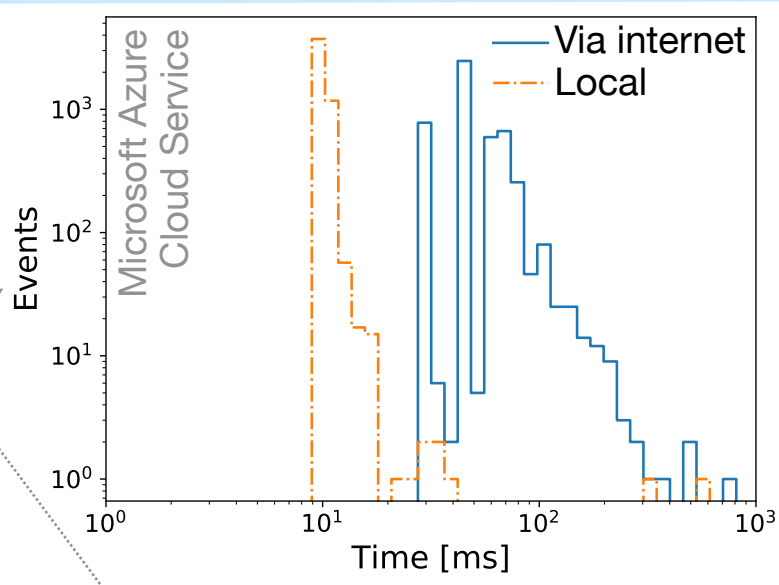

Top quark

\section{Results}

An FPGA-aaS reaches the same throughput as a locally connected GPU, the former by having many CPUs access it and the latter by setting a high batch size

- What NN architectures

are suitable for our

physics problems and laaS?
- How scalable are these solutions to HL-LHC data volumes?

룰 Fermilab UCSanDiego

GPU

batch size 1

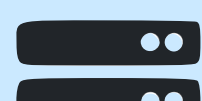

